\title{
Energy spectra of muonic atoms in quantum electrodynamics
}

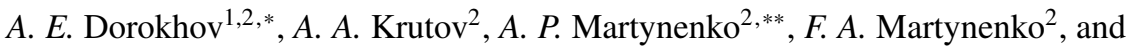 \\ O. S. Sukhorukova ${ }^{2}$ \\ ${ }^{1}$ Joint Institute for Nuclear Research, BLTP, 141980, Moscow region, Dubna, Russia \\ ${ }^{2}$ Samara University, 443086, Samara, Russia
}

\begin{abstract}
Vacuum polarization, nuclear structure and recoil, radiative corrections to the hyperfine structure of S-states in muonic ions of lithium, beryllium and boron are calculated on the basis of quasipotential method in quantum electrodynamics. We consider contributions in first and second orders of perturbation theory which have the order $\alpha^{5}$ and $\alpha^{6}$ in the energy spectrum. Total values of hyperfine splittings are obtained which can be used for a comparison with future experimental data.
\end{abstract}

\section{Introduction}

In 2010-2018 the CREMA (Charge Radius Experiments with Muonic Atoms) collaboration performed a number of experiments with muonic hydrogen and ions of muonic helium by methods of laser spectroscopy [1-4]. The measurement of the Lamb shift and hyperfine structure (HFS) of these muonic atoms led to new more precise values of charge radii of proton, deuteron, helion and $\alpha$-particle. So, as a result of measuring the transition frequency $2 P_{3 / 2}^{F=2}-2 S_{1 / 2}^{F=1}$ a more accurate value of the proton charge radius was found to be $r_{p}=$ $0.84184(67) \mathrm{fm}$, that differs from the value recommended by CODATA for $7 \sigma$ [5]. The CODATA value is based on the spectroscopy of the electronic hydrogen atom and on electronnucleon scattering. The measurement of the transition frequency $2 P_{3 / 2}^{F=1}-2 S_{1 / 2}^{F=0}$ for the singlet $2 S$ of the state $(\mu p)$ allowed one to obtain the hyperfine splitting of the $2 S$ energy level in muonic hydrogen, and also the values of the Zemach's radius $r_{Z}=1.082(37) \mathrm{fm}$ and magnetic radius $r_{M}=0.87(6) \mathrm{fm}$. The first measurement of three transition frequencies between energy levels $2 P$ and $2 S$ for muonic deuterium $\left(2 S_{1 / 2}^{F=3 / 2}-2 P_{3 / 2}^{F=5 / 2}\right),\left(2 S_{1 / 2}^{F=1 / 2}-\right.$ $\left.2 P_{3 / 2}^{F=3 / 2}\right),\left(2 S_{1 / 2}^{F=1 / 2}-2 P_{3 / 2}^{F=1 / 2}\right)$ allowed us to obtain a more accurate value (by 2.7 times) of the charge radius of the deuteron, that is also less than the value recommended by CODATA by $7.5 \sigma[3,5]$. As a result, the situation emerges when there is an inexplicable discrepancy between the values of such fundamental parameters, as the charge radius of a proton and deuteron, obtained from electronic and muonic atoms. The disagreement between theory and experiment [1-3] in the case of muonic hydrogen with the charge radii of proton and deuteron from [5] can be interpreted as an insufficiently accurate construction of the particle interaction operator, which manifests specifically for muonic atoms $[6,7]$. For different energy intervals,

\footnotetext{
*e-mail: dorokhov@ @heor.jinr.ru

**e-mail: a.p.martynenko@samsu.ru
} 
there are leading corrections related to the charge radius of the nucleus or the Zemach radii. Therefore, from experiments on the Lamb shift, we can obtain more accurate values of the nucleus charge radii, and on the hyperfine structure - the values of the magnetic radius and Zemach radius.

\section{General formalism}

Nuclear structure corrections in the HFS are determined by the electromagnetic form factors of the nuclei [8]. Among the nuclei which we consider, several nuclei have a spin $s_{2}=3 / 2$. The amplitude of the one-photon interaction of these nuclei with a muon can be written in the form [9]:

$$
\begin{gathered}
i M_{1 \gamma}=-\frac{Z e^{2}}{k^{2}}\left[\bar{u}\left(q_{1}\right) \gamma_{\mu} u\left(p_{1}\right)\right]\left[\bar{v}_{\alpha}\left(p_{2}\right) O_{\alpha \mu \beta} v_{\beta}\left(q_{2}\right)\right]=-\frac{Z e^{2}}{k^{2}}\left[\bar{u}\left(q_{1}\right) \gamma_{\mu} u\left(p_{1}\right)\right] \bar{v}_{\alpha}\left(p_{2}\right) \\
\left\{g_{\alpha \beta} \frac{\left(p_{2}+q_{2}\right)_{\mu}}{2 m_{2}} F_{1}\left(k^{2}\right)-g_{\alpha \beta} \sigma_{\mu \nu} \frac{k^{v}}{2 m_{2}} F_{2}\left(k^{2}\right)+\frac{k_{\alpha} k_{\beta}}{4 m_{2}^{2}} \frac{\left(p_{2}+q_{2}\right)_{\mu}}{2 m_{2}} F_{3}\left(k^{2}\right)-\frac{k_{\alpha} k_{\beta}}{4 m_{2}^{2}} \sigma_{\mu v} \frac{k^{v}}{2 m_{2}} F_{4}\left(k^{2}\right)\right\} v_{\beta}\left(q_{2}\right)
\end{gathered}
$$

where $p_{1}, p_{2}$ are four-momenta of particles in the initial state, $q_{1}, q_{2}$ are four-momenta of particles in the final state, $k=q_{2}-p_{2}=p_{1}-q_{1} . O_{\alpha \mu \beta}$ is the vertex function of the spin $3 / 2$ nucleus. Nuclei with a spin $3 / 2$ are described by the spin-vector $v_{\alpha}(p)$. Four form factors $F_{i}\left(k^{2}\right)$ are related to the charge $G_{E 0}$, electroquadrupole $G_{E 2}$, magnetic dipole $G_{M 1}$ and magnetic octupole $G_{M 3}$ form factors by the following expressions [9]:

$$
\begin{gathered}
G_{E 0}=\left(1+\frac{2}{3} \tau\right)\left[F_{1}+\tau\left(F_{1}-F_{2}\right)\right]-\frac{\tau}{3}(1+\tau)\left[F_{3}+\tau\left(F_{3}-F_{4}\right)\right] \\
G_{E 2}=F_{1}+\tau\left(F_{1}-F_{2}\right)-\frac{1+\tau}{2}\left[F_{3}+\tau\left(F_{3}-F_{4}\right)\right], \\
G_{M 1}=\left(1+\frac{4}{3} \tau\right) F_{2}-\frac{2}{3} \tau(1+\tau) F_{4}, \quad G_{M 3}=F_{2}-\frac{1}{2}(1+\tau) F_{4}, \quad \tau=-\frac{k^{2}}{4 m_{2}^{2}} .
\end{gathered}
$$

The leading order hyperfine splitting (the Fermi energy) can be obtained from the amplitude $M_{1 \gamma}$. When two moments are added, two states appear with the total angular momentum $F=2$ and $F=1$. To select the contribution of the amplitude $M_{1 \gamma}$ to the interaction operator of particles with $F=2$ and $F=1$, we use the method of projection operators, which are constructed from the wave functions of free particles in the rest frame $[10,11]$. Thus, the projection operator on a state with $F=2$ is equal to

$$
\hat{\Pi}_{\alpha}=\left[u(0) \bar{v}_{\alpha}\right]_{F=2}=\frac{1+\gamma_{0}}{2 \sqrt{2}} \gamma_{\beta} \varepsilon_{\alpha \beta},
$$

where the tensor $\varepsilon_{\alpha \beta}$ describes a muonic atom with $\mathrm{F}=2$. As a result, the projection of $M_{1 \gamma}$ to the state with $\mathrm{F}=2$ takes the form:

$$
\begin{gathered}
i M_{1 \gamma}(F=2)=-\frac{Z e^{2}}{16 k^{2} m_{1}^{2} m_{2}^{2}} \operatorname{Tr}\left\{( \hat { q } _ { 1 } + m _ { 1 } ) \gamma _ { \mu } ( \hat { p } _ { 1 } + m _ { 1 } ) \frac { 1 + \hat { v } } { 2 \sqrt { 2 } } \gamma _ { \rho } \varepsilon _ { \alpha \rho } ( \hat { p } _ { 2 } - m _ { 2 } ) \left[g_{\alpha \beta} \frac{\left(p_{2}+q_{2}\right)_{\mu}}{2 m_{2}} F_{1}\left(k^{2}\right)\right.\right. \\
\left.\left.-g_{\alpha \beta} \sigma_{\mu v} \frac{k^{v}}{2 m_{2}} F_{2}\left(k^{2}\right)+\frac{k_{\alpha} k_{\beta}}{4 m_{2}^{2}} \frac{\left(p_{2}+q_{2}\right)_{\mu}}{2 m_{2}} F_{3}\left(k^{2}\right)-\frac{k_{\alpha} k_{\beta}}{4 m_{2}^{2}} \sigma_{\mu v} \frac{k^{v}}{2 m_{2}} F_{4}\left(k^{2}\right)\right]\left(\hat{q}_{2}-m_{2}\right) \gamma_{\lambda} \frac{1+\hat{v}}{2 \sqrt{2}} \varepsilon_{\beta \lambda}^{*}\right\},
\end{gathered}
$$

where auxiliary four-vector $v=(1,0,0,0)$. 
Table 1. Hyperfine splittings of $S$-states in muonic ions $\left(\mu_{3}^{6} L i\right)^{2+}$ and $\left(\mu_{3}^{7} L i\right)^{2+}$

\begin{tabular}{|c|c|c|c|c|c|}
\hline No. & Contribution to the splitting & \multicolumn{2}{|c|}{$\left(\mu_{3}^{6} L i\right)^{2+}, \mathrm{meV}$} & \multicolumn{2}{|c|}{$\left(\mu_{3}^{7} \mathrm{Li}\right)^{2+}, \mathrm{meV}$} \\
\hline & & $1 \mathrm{~S}$ & $2 \mathrm{~S}$ & $1 \mathrm{~S}$ & $2 \mathrm{~S}$ \\
\hline 1 & $\begin{array}{l}\text { Contribution of order } \alpha^{4}, \\
\text { the Fermi energy }\end{array}$ & 1416.07 & 177.01 & 5026.00 & 628.25 \\
\hline 2 & Muon AMM contribution & 1.65 & 0.21 & 5.87 & 0.73 \\
\hline 3 & $\begin{array}{l}\text { Relativistic correction } \\
\text { of order } \alpha^{6}\end{array}$ & 1.02 & 0.18 & 3.62 & 0.64 \\
\hline 4 & $\begin{array}{l}\text { Nuclear structure } \\
\text { correction of order } \alpha^{5}\end{array}$ & $\begin{array}{l}\text { G: }-109.92 \\
\mathrm{U}:-112.02\end{array}$ & $\begin{array}{l}\text { G: }-13.74 \\
\text { U: }-14.00\end{array}$ & $\begin{array}{l}\text { G: }-369.25 \\
U:-376.31\end{array}$ & $\begin{array}{l}\text { G: }-46.16 \\
\text { U: }-47.04\end{array}$ \\
\hline 5 & Nuclear structure and recoil & G: -0.20 & G: -0.03 & G: -30.67 & G: -3.83 \\
\hline 6 & $\begin{array}{l}\text { Nuclear structure correction } \\
\text { of order } \alpha^{6} \text { in } 1 \gamma \text { interaction }\end{array}$ & 3.35 & 0.34 & 10.67 & 1.08 \\
\hline 7 & $\begin{array}{l}\text { Nuclear structure correction } \\
\text { in the second order of PT }\end{array}$ & -2.56 & -0.90 & -8.19 & -2.90 \\
\hline 8 & $\begin{array}{l}\text { VP correction of order } \\
\alpha^{5} \text { in the first order of PT }\end{array}$ & 5.22 & 0.67 & 18.54 & 2.38 \\
\hline 9 & $\begin{array}{c}\text { VP correction of order } \\
\alpha^{5} \text { in the second order of PT }\end{array}$ & 12.05 & 1.11 & 42.83 & 3.94 \\
\hline 10 & $\begin{array}{c}\text { Muon VP correction of } \\
\text { order } \alpha^{6} \text { in the first order of PT }\end{array}$ & 0.08 & 0.01 & 0.29 & 0.04 \\
\hline 11 & $\begin{array}{c}\text { Muon VP correction of } \\
\text { order } \alpha^{6} \text { in the second order of PT }\end{array}$ & 0.09 & 0.01 & 0.31 & 0.04 \\
\hline 12 & $\begin{array}{l}\text { VP correction of order } \\
\alpha^{6} \text { in the first order of PT }\end{array}$ & 0.07 & 0.01 & 0.24 & 0.03 \\
\hline 13 & $\begin{array}{c}\text { VP correction of order } \\
\alpha^{6} \text { in the second order of PT }\end{array}$ & 0.14 & 0.02 & 0.53 & 0.05 \\
\hline 14 & $\begin{array}{l}\text { Nuclear structure and VP } \\
\text { correction of order } \alpha^{6}\end{array}$ & -1.62 & -0.20 & -5.85 & -0.73 \\
\hline 15 & $\begin{array}{l}\text { Nuclear structure and muon } \\
\text { VP correction of order } \alpha^{6}\end{array}$ & -0.14 & -0.02 & -0.51 & -0.06 \\
\hline 16 & $\begin{array}{l}\text { Hadron VP correction } \\
\text { of order } \alpha^{6}\end{array}$ & 0.06 & 0.01 & 0.21 & 0.03 \\
\hline 17 & $\begin{array}{c}\text { Radiative nuclear finite } \\
\text { size correction of order } \alpha^{6}\end{array}$ & -0.34 & -0.04 & -1.24 & -0.15 \\
\hline & Summary contribution & 1325.02 & 164.65 & 4693.40 & 583.38 \\
\hline
\end{tabular}

To introduce the projection operators for another state of hyperfine structure with $F=1$, we use the following expansion:

$$
\Psi_{S_{2}=3 / 2, F=1, F_{z}}=\sqrt{\frac{2}{3}} \Psi_{S=0, F=1, F_{z}}+\frac{1}{\sqrt{3}} \Psi_{S=1, F=1, F_{z}},
$$

where the Rarita-Schwinger spinor $v_{\alpha}(p)$ for the state with $s_{2}=3 / 2$ is presented as a result of adding spin $1 / 2$ and angular momentum 1 . With this method of adding moments, the total spin $S$ can take two values $S=1$ and $S=0$. When calculating the matrix elements for the states $\Psi_{01 F_{z}}$ and $\Psi_{11 F_{z}}$, we successively perform the projection on the state with spin $S=0$, $S=1$, and then on the state with the total angular momentum $F=1$. The corresponding 
projection operators have the form:

$$
\hat{\Pi}_{\alpha}(S=0, F=1)=\frac{1+\hat{v}}{2 \sqrt{2}} \gamma_{5} \varepsilon_{\alpha}, \hat{\Pi}_{\alpha}(S=1, F=1)=\frac{1+\hat{v}}{4} \gamma_{\sigma} \varepsilon_{\alpha \sigma \rho \omega} v^{\rho} \varepsilon^{\omega}
$$

where $\varepsilon^{\omega}$ is the polarization vector of the state with $\mathrm{F}=1$. Using (5)-(6) we obtain the following value of the hyperfine splitting (the Fermi energy):

$$
\Delta E_{1 \gamma}^{h f s}=E_{F}(n S)=\frac{16}{9} \frac{\pi Z \alpha}{m_{1} m_{2}} F_{2}(0) \frac{W^{3}}{\pi n^{3}}=\frac{16 \alpha(Z \alpha)^{3} \mu^{3}}{9 m_{1} m_{p} n^{3}} \mu_{N},
$$

with $W=\mu Z \alpha$.

Acting as in the case of the one-photon interaction, we can present the contribution of two-photon exchange interactions to HFS in the form:

$$
\begin{gathered}
\Delta E^{h f s}(n S)=\left|\psi_{n S}(0)\right|^{2} \int d^{4} k V_{2 \gamma}(k)=\frac{64}{9} \frac{(Z \alpha)^{2}}{\pi^{2}}\left|\psi_{n S}(0)\right|^{2} \int \frac{d^{4} k}{k^{4}\left(k^{4}+4 m_{1}^{2} k_{0}^{2}\right)\left(k^{4}+4 m_{2}^{2} k_{0}^{2}\right)} \times \\
{\left[F_{1} F_{2}\left(k^{6}-k^{4} k_{0}^{2}+\frac{4}{15} \frac{k^{4} k_{0}^{4}}{m_{2}^{2}}-\frac{7}{10} \frac{k^{6} k_{0}^{2}}{m_{2}^{2}}+\frac{13}{30} \frac{k^{8}}{m_{2}^{2}}\right)+F_{2} F_{4}\left(-\frac{1}{30} \frac{k^{2} k_{0}^{6}}{m_{2}^{2}}+\frac{1}{15} \frac{k^{4} k_{0}^{4}}{m_{2}^{2}}-\frac{1}{30} \frac{k^{6} k_{0}^{2}}{m_{2}^{2}}\right)+\right.} \\
F_{2} F_{3}\left(-\frac{1}{15} \frac{k^{2} k_{0}^{6}}{m_{2}^{2}}+\frac{11}{60} \frac{k^{4} k_{0}^{4}}{m_{2}^{2}}-\frac{7}{60} \frac{k^{8}}{m_{2}^{2}}\right)+F_{1} F_{4}\left(-\frac{1}{5} \frac{k^{2} k_{0}^{6}}{m_{2}^{2}}+\frac{3}{10} \frac{k^{4} k_{0}^{4}}{m_{2}^{2}}-\frac{1}{10} \frac{k^{8}}{m_{2}^{2}}\right)+ \\
\left.F_{2}^{2}\left(\frac{1}{15} \frac{k^{2} k_{0}^{6}}{m_{2}^{2}}-\frac{1}{6} k^{2} k_{0}^{4}-\frac{2}{15} \frac{k^{4} k_{0}^{4}}{m_{2}^{2}}+\frac{1}{6} k^{4} k_{0}^{2}+\frac{23}{120} \frac{k^{6} k_{0}^{2}}{m_{2}^{2}}-\frac{1}{4} \frac{k^{8}}{m_{2}^{2}}\right)\right] .
\end{gathered}
$$

In (8) we have the Zemach and recoil corrections. The form factors $F_{i}\left(k^{2}\right)$ are expressed in terms of $G_{E 0}, G_{E 2}, G_{M 1}, G_{M 3}$ for which the Gaussian parametrization is used in numerical calculations of integrals with respect to $\mathrm{k}$. The values of the form factors at zero have the form [13]:

$$
G_{E 0}(0)=1, \quad G_{M 1}(0)=\frac{m_{2} \mu_{N}}{m_{p} Z}, \quad G_{E 2}(0)=m_{2}^{2} Q, \quad G_{M 3}(0)=\frac{m_{2}}{m_{p} Z} m_{2}^{2} \Omega .
$$

Various parameters of light nucleus ( $\mathrm{Li}, \mathrm{Be}, \mathrm{B})$ were investigated in electron scattering experiments $[12,13]$. Another correction for the structure of the nucleus of order $\alpha^{6}$ is obtained as a result of an expansion of magnetic form factor in the first order of perturbation theory (PT) and electric form factor in the second order of PT. The contribution to the HFS in this case has the form ( $R$ is the radius of uniformly charged sphere, $R=\sqrt{5} r_{N} / \sqrt{3}, r_{N}$ is the nucleus charge radius, magnetic radius $r_{M} \approx r_{N}$ ):

$$
\begin{gathered}
\Delta E_{1 \gamma, s t r}^{h f s}=\frac{2}{3} \mu^{2} Z^{2} \alpha^{2} r_{M}^{2} \frac{3 n^{2}+1}{n^{2}} E_{F}(n S), \\
\Delta E_{\text {str,sopt }}^{h f s}(1 S)=E_{F}(1 S) \frac{R^{2} W^{2}}{4}\left[\frac{4}{75}(-53+15 C+15 \ln R W)-\frac{R W}{12}(-15+4 C+4 \ln R W)\right], \\
\Delta E_{\text {str,sopt }}^{h f s}(2 S)=E_{F}(2 S) \frac{R^{2} W^{2}}{4}\left[\frac{4}{75}(-107+60 C+60 \ln R W)+\frac{R W}{3}(17-8 C-8 \ln R W)\right],
\end{gathered}
$$

where an expansion in $(R W)$ up to terms of the first order in square brackets is used $\left(R W\left({ }_{3}^{6} \mathrm{Li}\right)=0.038, R W\left({ }_{3}^{7} \mathrm{Li}\right)=0.036, R W\left({ }_{4}^{9} \mathrm{Be}\right)=0.050, R W\left({ }_{5}^{10} \mathrm{~B}\right)=0.060, R W\left({ }_{5}^{11} \mathrm{~B}\right)=\right.$ $0.060)$. 


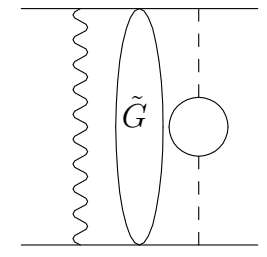

a

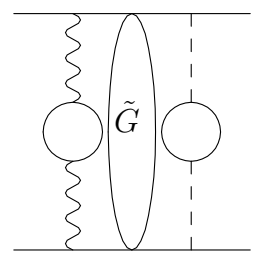

$\mathrm{b}$

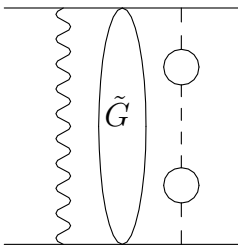

C

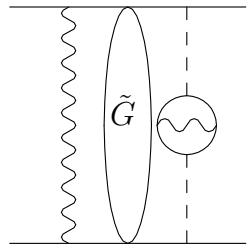

d

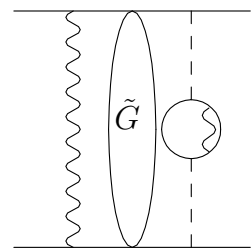

e

Figure 1. Effects of one- and two-loop vacuum polarization in the second order of PT

Another important corrections are connected with vacuum polarization (VP) effects. To calculate a contribution of one-loop VP in the first order of PT, we use the following potential:

$$
\Delta V_{1 \gamma, v p}^{h f s}(r)=\frac{4 \alpha g_{N}\left(1+a_{\mu}\right)}{3 m_{1} m_{p}}\left(\mathbf{s}_{1} \mathbf{s}_{2}\right) \frac{\alpha}{3 \pi} \int_{1}^{\infty} \rho(\xi) d \xi\left(\pi \delta(\mathbf{r})-\frac{m_{e}^{2} \xi^{2}}{r} e^{-2 m_{e} \xi r}\right),
$$

where $g_{N}=\mu_{N} / s_{2}$, spectral function $\rho(\xi)=\sqrt{\xi^{2}-1}\left(2 \xi^{2}+1\right) / \xi^{4}$. Averaging (13) over $S$ wave functions we obtain the contribution of order $\alpha^{5}$ to HFS (see numerical results in Tab. 1 in the case of muonic lithium). The main contribution of the electron VP to HFS in the second order of PT (sopt) has the form (see Fig. 1(a)):

$$
\Delta E_{\text {sopt vp } 1}^{\text {hfs }}=2<\psi\left|\Delta V_{V P}^{C} \cdot \tilde{G} \cdot \Delta V_{B}^{h f s}\right| \psi>
$$

where the Coulomb potential, modified by the one-loop VP effect, is the following:

$$
\Delta V_{v p}^{C}(r)=\frac{\alpha}{3 \pi} \int_{1}^{\infty} \rho(\xi) d \xi\left(-\frac{Z \alpha}{r}\right) e^{-2 m_{e} \xi r}
$$

More complicate two-loop VP corrections are determined by potentials in the first order of PT [14]:

$$
\begin{gathered}
\Delta V_{V P-V P}^{C}(r)=\left(\frac{\alpha}{3 \pi}\right)^{2} \int_{1}^{\infty} \rho(\xi) d \xi \int_{1}^{\infty} \rho(\eta) d \eta\left(-\frac{Z \alpha}{r}\right) \frac{1}{\xi^{2}-\eta^{2}}\left(\xi^{2} e^{-2 m_{e} \xi r}-\eta^{2} e^{-2 m_{e} \eta r}\right), \\
\Delta V_{2-\text { loop } V P}^{C}(r)=-\frac{2 Z \alpha^{3}}{3 \pi^{2} r} \int_{0}^{1} \frac{f(v) d v}{\left(1-v^{2}\right)} e^{-\frac{2 m_{e} r}{\sqrt{1-v^{2}}}} .
\end{gathered}
$$

The two-loop VP corrections in the second order of PT are presented in Figs. 1(b,c,d,e). Omitting intermediate expressions we include corresponding numerical results in Tab. 1.

One-loop VP correction is also determined by two photon exchange amplitudes in Fig. 2. To calculate it, it is necessary to use the potential $V_{2 \gamma}(k)$ from (8), modifying it accordingly. 


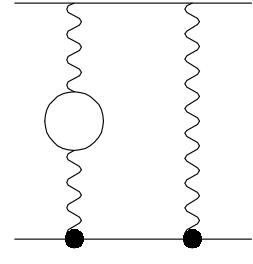

a

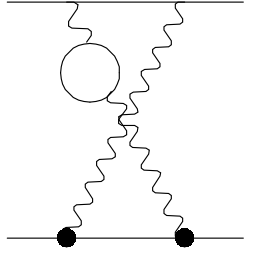

b

Figure 2. Two photon exchange amplitudes accounting for effects of vacuum polarization and nuclear structure

As a result, the contribution to the HFS spectrum is equal to the following:

$$
E_{2 \gamma, v p}^{h f s}=-\frac{2 \mu^{3} Z^{3} \alpha^{4}}{9 \pi^{2} n^{3}} \int \frac{V_{2 \gamma}(k) d^{4} k}{k^{3}}\left[5 k^{3}-12 m_{e} k^{2}-6\left(k^{2}-2 m_{e}^{2}\right) \sqrt{k^{2}+4 m_{e}^{2}} \operatorname{Arcth}\left[\frac{k}{\sqrt{k^{2}+4 m_{e}^{2}}}\right] .\right.
$$

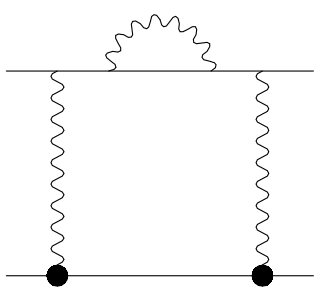

a

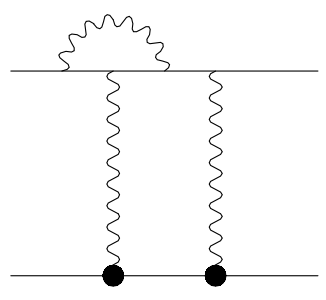

$\mathrm{b}$

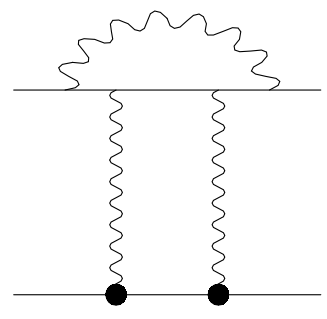

c

Figure 3. Direct two-photon exchange amplitudes with radiative corrections to muon line giving contributions of order $E_{F} \alpha(Z \alpha)$ to the hyperfine structure

Finally, it is also necessary to take into account the radiative correction with the nuclear structure, which is determined by three amplitudes in Fig. 3 [15, 16]. For them, integral expressions are obtained in $[10,15]$ that can be used to find numerical results (see Tab. 1).

\section{Conclusion}

In this work we continue investigations [17] of the energy spectra of light muonic atoms regarding the HFS. An estimate of basic corrections for muonic ions of lithium, beryllium and boron was done in [18]. Numerical results for different corrections in HFS are presented in Tab. 1 for ions of muonic lithium. Total values of HFS in the case of ions of muonic beryllium and boron are the following: $-4080.71 \mathrm{meV}\left(\left(\mu_{4}^{9} B e\right)^{3+}, 1 \mathrm{~S}\right),-505.82 \mathrm{meV}\left(\left(\mu_{4}^{9} B e\right)^{3+}, 2 \mathrm{~S}\right)$; $10233.86 \mathrm{meV}\left(\left(\mu_{5}^{10} B\right)^{4+}, 1 \mathrm{~S}\right), 1265.03 \mathrm{meV}\left(\left(\mu_{5}^{10} B\right)^{4+}, 2 \mathrm{~S}\right), 17572.70 \mathrm{meV}\left(\left(\mu_{5}^{11} B\right)^{4+}, 1 \mathrm{~S}\right)$, $2172.56 \mathrm{meV}\left(\left(\mu_{5}^{11} B\right)^{4+}, 2 \mathrm{~S}\right)$. The main theoretical uncertainty of the calculations is due to the measurement errors of the nuclear form factors in the kinematically important region of momenta and polarizability correction [19]. It amounts to about 1 percent.

The work is supported by Russian Science Foundation (grant No. RSF 18-12-00128). 


\section{References}

[1] A. Antognini et al., Science 339, 417 (2013)

[2] A. Antognini et al., Ann. Phys. (NY) 331, 127 (2013)

[3] R. Pohl, F. Nez, L. M. P. Fernandes et al., Science 353, 669 (2016)

[4] M. Diepold, B. Franke, J. J. Krauth et al., Ann. Phys. 396, 220 (2018)

[5] P. J. Mohr, D. B. Newell and B. N. Taylor, Rev. Mod. Phys. 88, 035009 (2016)

[6] A. E. Dorokhov et al., Phys. Part. Nucl. Lett. 14, 857 (2017)

[7] A. E. Dorokhov et al., Phys. Lett. B 776, 105 (2018)

[8] R. N. Faustov and A. P. Martynenko, Phys. Rev. A 67, 052506 (2003)

[9] S. Nozawa and D. B. Leinweber, Phys. Rev. D 42, 3567 (1990)

[10] A. P. Martynenko, F. A. Martynenko and R. N. Faustov, JETP 124, 895 (2017)

[11] A. E. Dorokhov et al., Eur. Phys. Jour. A 54, 131 (2018)

[12] H. Uberall, Electron scattering from complex nuclei (Acad. press, NY, London, 1971)

[13] G. H. Fuller, Jour. Phys. and Chem. Ref. Data 5, 835 (1976)

[14] R. N. Faustov et al., Phys. Rev. A 90, 012520 (2014)

[15] R. N. Faustov et al., Phys. Lett. B 733, 354 (2014)

[16] R. N. Faustov et al., Phys. Lett. B 775, 79 (2017)

[17] A. A. Krutov et al., Phys. Rev. A 94, 062505 (2016)

[18] R. Swainson and G. W. F. Drake, Phys. Rev. A 34, 620 (1986)

[19] K. Pachucki, Phys. Rev. A 76, 022508 (2007) 